\title{
Application of Poly(vinylidene fluoride) and Poly(methyl methacrylate) Blends to Optical Material
}

\author{
Akira Tanaka, Hisashi Sawada, and Yuji KoJima \\ Fujitsu Limited, 1015, Kamikodanaka, Nakahara-ku, Kawasaki 211, Japan
}

(Received December 1, 1989)

\begin{abstract}
Physical properties such as light transmittance, deflection temperature, elongation, impact strength, and melting point have been studied for poly(vinylidene fluoride) (PVDF) and poly(methyl methacrylate) (PMMA) blends in relation to applications to optical components. The blends were found to be transparent for PVDF concentrations up to $80 \mathrm{wt} \%$ due to the limited size of the spherulites of PVDF smaller than the visual wavelength. Maximum elongation is given at a PVDF concentration of $70 \mathrm{wt} \%$. The deflection temperature is minimum at a PVDF concentration of $50 \%$. From these results, it is concluded that the blend with $70 \%$ PVDF is most suitable for optical applications such as cladding material for optical fiber and birefringent film for super twisted nematic liquid crystal displays.

KEY WORDS Poly(vinylidene fluoride) / Polymer Alloy / Poly(methyl methacrylate) / Optical Fiber / Liquid Crystal / Birefringent Film / Cladding /
\end{abstract}

Poly(methyl methacrylate) (PMMA) is widely used in optical components such as fibers, lenses, and discs, because of its transparency and hardness. However, PMMA is difficult to mold into a thin film because it is brittle in a glassy state.

Poly(vinylidene fluoride) (PVDF) is superior to PMMA in terms of mechanical strength and environmental characteristics. It also has high dielectric and piezoelectric constants associated with the large dipole moment of the carbon -fluorine bond, and their alignment to the $b$ axis. PVDF is used in electrical and acoustic components such as condensors and piezoelectric film. However, PVDF is not suitable for use as optical materials because it has poor transparency. Polymer blends of PMMA and PVDF have lower critical solution temperatures' and good transparency. ${ }^{2}$ Harn and Wendorff ${ }^{3}$ reported that the blend containing about $17 \mathrm{wt} \%$ PVDF has zero birefringence. This suggests that this blend is suitable as an optical material. The PVDF crystal, especially in the $\beta$ form, which has a large dipole moment, is expected to have a large birefringence. That would make the blend applicable as a birefringent film control polarized light.

This paper describes the physical properties of this blend and application to optical fibers with polycarbonate (PC) as core material, ${ }^{4}$ and as birefringent film for black and white super twisted nematic (STN) liquid crystal displays (LCD).

\section{EXPERIMENTAL}

PMMA resin was obtained from Kurare Chemical Industry (Palaglass). PVDF resin was obtained from Kureha Chemical Industry (KF-polymer). They were melt-blended in an extruder to ensure homogeneous mixing and dried in a heat chamber before processing. Thin films about $150 \mu \mathrm{m}$ thick were extruded from the blends by the T-dies. ${ }^{5}$ Birefringent films were uniaxially stretched up to different retardations $\left(\Delta n_{\mathrm{d}}\right)$ at elevated temperatures, using the tenter method. Some samples were then annealed. Also sheets of $3.3 \mathrm{~mm}$ thick were prepared in a hot press. Optical fiber was spun into a filament by forcing the molten PC and 
the polymer blend of PMMA and PVDF through double nozzles near the melting point. The fiber thickness was maintained at $1 \mathrm{~mm}$ by controlling the drive velocity. ${ }^{5}$

The light transmittance was measured with a spectrophotometer. The refractive index was determined by Abbe's method. The thermal behavior was observed with a differential scanning calorimeter. The deflection temperature was measured by the ASTM 1527-65T test, and elongation until breake was measured based on JIS-K 7113. The impact strength was measured using a dyne statt method. The optical birefringence was determined with a polarizing microscope using a Berek compensator. The contrast and chromaticity were measured with a chromaticity meter from the panel of the an STN LCD made by Fujitsu Limited. The back-light was a cold-cathode fluorescent lamp.

\section{RESULTS AND DISCUSSION}

\section{Light Transmission}

Figure 1 shows the dependence of light transmisson of PVDF/PMMA blend on composition. For PVDF weight percentage of less than $75 \%$, the optical transmission is constant at about $92 \%$. When the PVDF concentration goes above $75 \%$, the transparency rapidly decreases. The refractive indexes of crystalline and amorphous regions for PVDF are 1.48 and $1.37 .^{3}$ Thus, the pure PVDF solid is not transparent because the crystalline and amorphous phase dimensions are larger than the wavelength of light due to Rayleigh scattering. However, in blends containing less than $75 \%$ PVDF, the phase dimensions of PVDF and PMMA are well below the wavelength of light. The refractive index of amorphous PMMA is 1.49 , nearly equal to that of PVDF crystal. This reduces light scattering in partially crystalline PVDF/ PMMA blends, improving their transparency significantly over pure PVDF. Horibe et al. ${ }^{6}$ reported that the transmittance at the ultraviolet

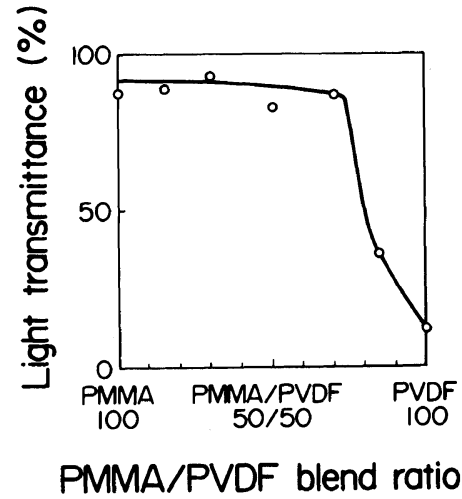

Figure 1. PMMA/PVDF blend ratio and light transmittance.

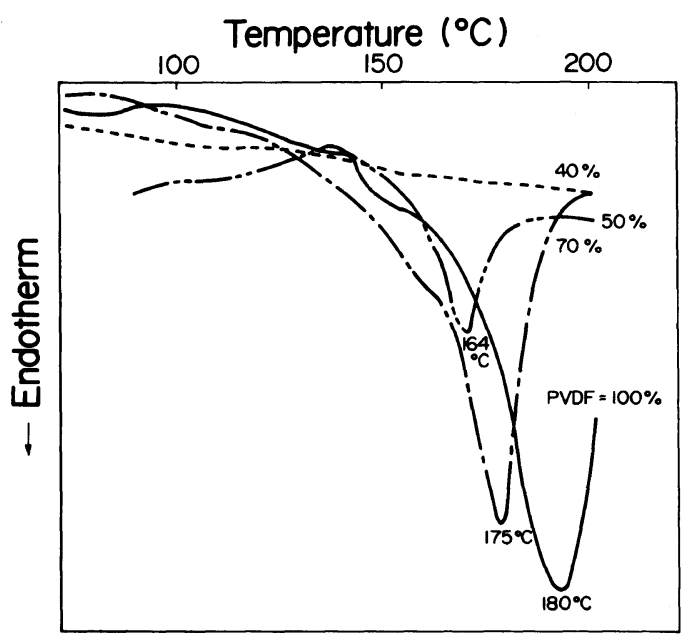

Figure 2. DSC thermograms for different concentrations of PMMA/PVDF blend.

wavelength $(265 \mathrm{~nm})$ of quenched samples of a melted $70 \%$ PVDF blend did not change after annealing, and that the crystal form of PVDF was in the $\beta$ phese in the $70 \%$ PVDF blend. Furthermore, the $\beta$-spherulites of PVDF did not grow larger than the wavelength of light, while the $\alpha$-spherulites grew to the order of $10 \mu \mathrm{m}^{4,7}$ We conclude that the blend with a PVDF weight percentage of $70 \%$ is transparent and thermally stable.

\section{Thermal Properties}

Figure 2 shows thermal behavior around the 
Temperature $\left({ }^{\circ} \mathrm{C}\right)$

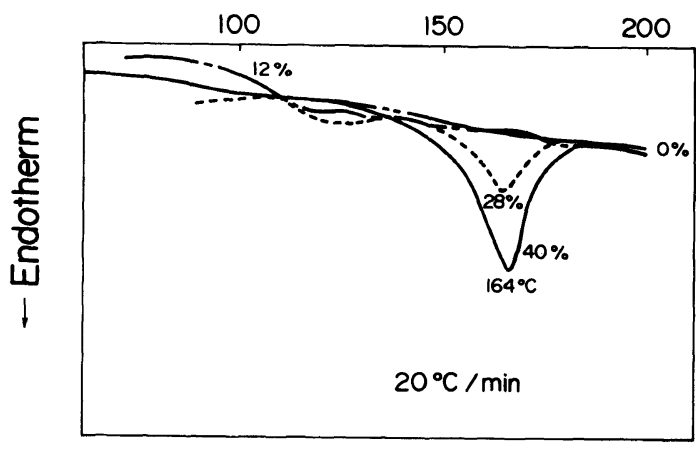

Figure 3. DSC thermograms for different concentrations of PMMA/PVDF blend after annealing.

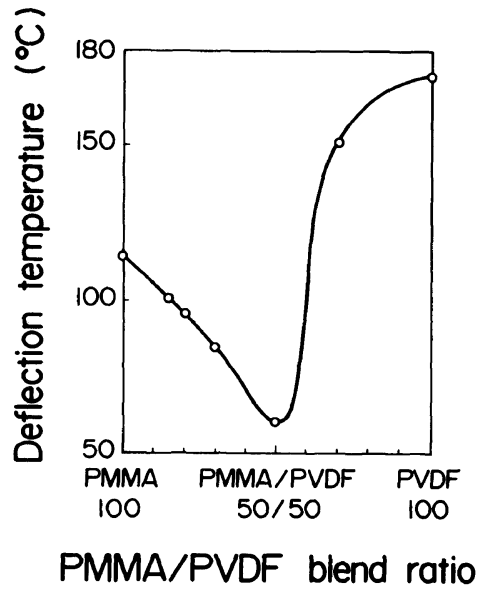

Figure 4. PMMA/PVDF blend ratio and deflection temperature.

melting point of these blends during heating at $20^{\circ} \mathrm{C} \mathrm{min}{ }^{-1}$. The melting point can not be observed for PVDF concentrations of less than $40 \%$. However, the melting point of the blend at $28 \%$ PVDF appears after annealing for $1 \mathrm{~h}$ at $110^{\circ} \mathrm{C}$ above the glass transition temperature of PMMA $\left(100^{\circ} \mathrm{C}\right)$, as is shown in Figure 3 .

Figure 4 shows the relationship between the deflection temperature and the blend ratio. The minimum point is observed at a ratio of $50 / 50$. The deflection temperature above $65 \%$ PVDF is higher than that of pure PMMA. This suggests that PVDF blends between $65 \%$ and $75 \%$ have almost the same transparency and higher deflection temperatures compared with

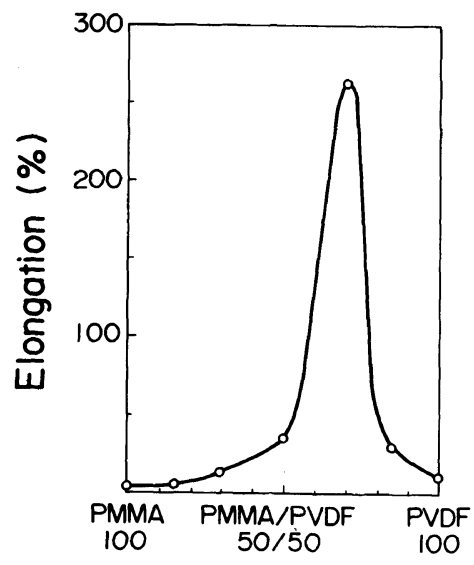

PMMA/PVDF blend ratio

Figure 5. PMMA/PVDF blend ratio and elongation up to breakage.

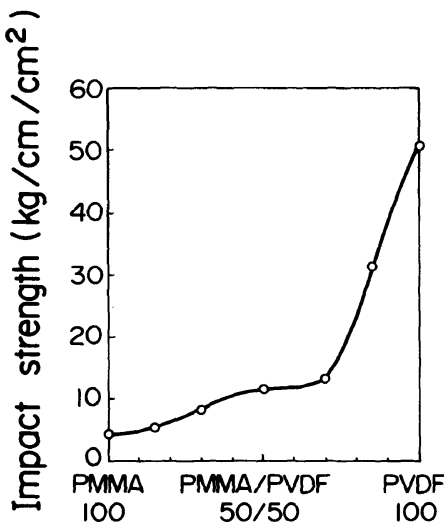

\section{PMMA/PVDF blend ratio}

Figure 6. PMMA/PVDF blend ratio and impact strength.

pure PMMA.

\section{Mechanical Properties}

Figure 5 shows elongation until break as a function of the blend ratio. The maximum point occurs at $70 \%$ PVDF where crystal-phase PVDF, amorphous-phase PVDF and PMMA make a homogeneous mixture. All have about the same mole fraction, and mix perfectly with each other. Figure 6 shows the relationship between the impact strength and the blend 
ratio. The impact strength decreases rapidly as the weight percentage PMMA increases up to $30 \%$. This is the reverse of the result for light transmittance (Figure 1). This small percentage of PMMA strongly affects the optical transmittance and mechanical strength. The texture size effects on these properties are found to be almost the same. Based on these results, we selected an optical thin film with a PVDF weight percentage of $70 \%$ as a cladding for fiber and birefringent film using an STNLCD.

\section{APPLICATIONS}

\section{Optical Fiber}

Plastic optical fiber consists of a core which transmits light and cladding. The interface between the core and cladding reflects light. The refractive index, $n_{2}$, of the cladding must be lower than that of the core, $n_{1}$, and transprent to decrease optical loss at the interface.

Figure 7 shows the structure of the plastic fiber with PC as the core. The PVDF/PMMA blend with a PVDF weight percentage of $70 \%$ is suitable as cladding material for the following reasons:

1. The blend is transparent, adhesive, and has good elongation, and thermal stability as good as that of PC.

2. The refractive index of the blend (1.445) is lower than that of PC (1.586). The numerical apertue is 0.65 . That of commercial PMMA core fiber is only $0.50 .^{8}$

Figure 8 is a cross section of the fabricated ffber. The blend, $10 \mu \mathrm{m}$ thick, is uniformly clad around the core. Adhesion is tested by continuous back and forth bending. The bending angle is $\pm 180^{\circ}$, and the bending radius $5 \mathrm{~mm}$. The fiber is coated by Santoprene, polypropylene commercialized by Monsanto Corp. Figure 9 shows the optical transmission loss at a wavelength of $660 \mathrm{~nm}$ as a function of the number of bendings. The loss of the

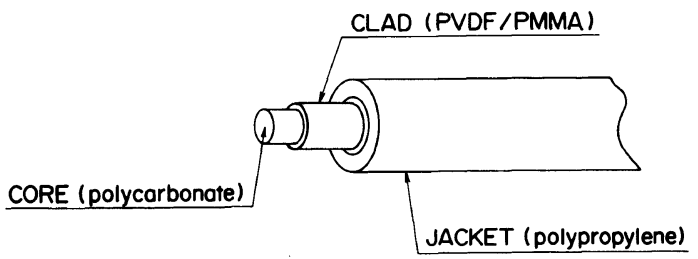

Figure 7. Optical fiber structure.

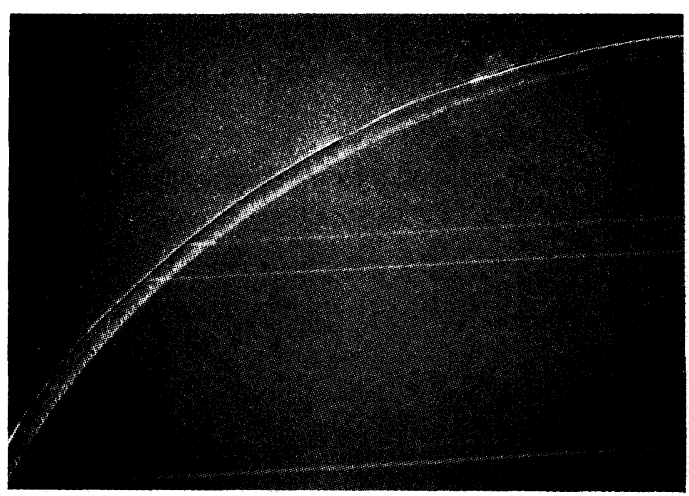

Figure 8. Cross section $(\times 300)$.

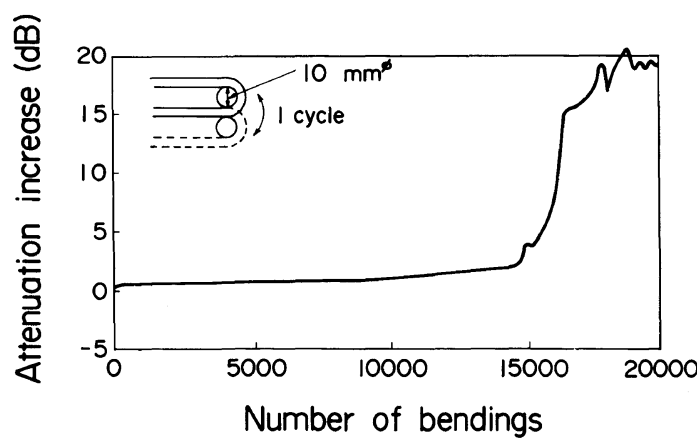

Figure 9. Increase of attenuation vs. number of continuous bendings.

fabricated fiber is stable until it is bent about $1.5 \times 10^{4}$ times. We expect that adhesion of the core and cladding materials is strong and this blend applicable for cladding. ${ }^{9}$ Elongation is also satisfactory for developing bending characteristics and adhesion.

\section{Birefringent Film for STN LCDs}

STN LCD, because of its high multiplexability, is widely used for application, such 
as word processors and hand-held computers where large information capacity is required. However, one of the problems with the STN LCD display is that the background colors are yellow or blue. The bakcground color of STN LCD is determined by the direction of the polarizer and the wavelength dependence of the light passing through the liquid crystal layer. To reduce the color change, we must control the polarization of oblique incident light before it reaches to the polarizer. We use such LCDs in double panels of LCDs, called DSTN $\mathrm{LCDs}^{10}$ to display black and white. DSTN LCD is heavy, thick, and expensive. To improve LCD, we placed the birefrngent film, usually of polycarbonate, ${ }^{10}$ on the conventional STN LCD to control polarization. ${ }^{11}$

To obtain different retardations $\left(\Delta n_{\mathrm{d}}\right)$, we elongated a blend film of $70 \mathrm{wt} \%$ PVDF uniaxially and annealed them near $100^{\circ} \mathrm{C} .{ }^{5}$ The film is about $150 \mu \mathrm{m}$ thick and has a light transmittance of $94 \%$. Figure 10 shows the contrast ratio as a function of $\Delta n$ d for the STN LCD panel made by Fujitsu using a coldcathode fluorecent lamp. The maximum contrast ratio of 7.8 is near $600 \mathrm{~nm}$, almost the same as that reported by Nagae et al. ${ }^{12}$ According to the CIE chromaticity diagram, $\mathrm{X}$ is 0.322 and $\mathrm{Y} 0.355$ when the background is white. $\mathrm{X}$ is 0.232 and $\mathrm{Y} 0.231$ when the background is black. Although the black mode is somewhat bluish, this blend is usable for birefringent film.

\section{CONCLUSIONS}

PVDF and PMMA polymer blends are transparent for PVDF concentrations up to $80 \%$. The maximum elongation is at $70 \%$ PVDF. The deflection temperature is minimum at $50 \%$ PVDF. A PVDF blend of $70 \mathrm{wt} \%$ is most stable for thin film in terms of transparency, thermal stabillity, and mechanical strength. These charactericstics are strongly affected by the $\beta$ PVDF crystal, which

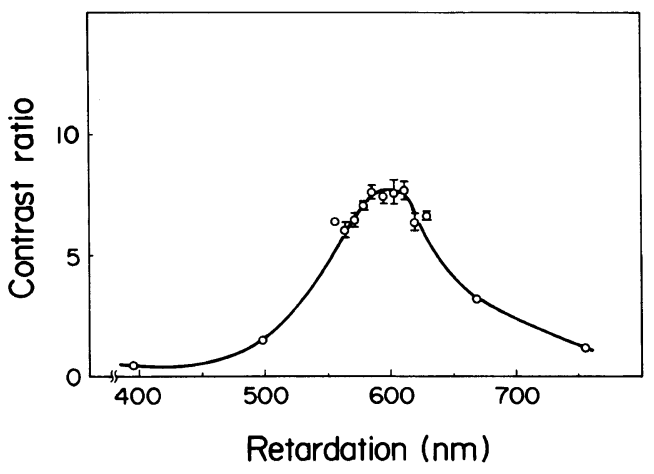

Figure 10. Retardation $\Delta n_{\mathrm{d}}$ of birefringent film and contrast ratio.

has spherulites smaller than the visual wavelength. This blend can be used as cladding for optical fiber and as birefringent film for super twisted nematic liquid crystal displays.

Acknowledgment. We thank the Kureha Chemical Industry staff for making the birefringent films and measuring the polymer blends.

\section{REFERENCES}

1. T. Nishi and T. T. Wang, Macromolecules, 8, 603 (1975).

2. Fujitsu, Japan Pagent 63-136105 (1988); 1-6422 (1989).

3. B. R. Hahn and J. H. Wendorff, Polymer, 26, 1619 (1985).

4. A. Tanaka, E. Takahashi, and N. Wakatsuki, Optical Fiber Communication, 38 (1986).

5. Kureha Chemical Industry and Fujitsu, Japan Patent 1-58295 (1989).

6. H. Horibe, F. Baba, and S. Itoh, Polym. Prepr., Jpn., 38 , 3533 (1989).

7. A. J. Lovinger, J. Polym. Sci., Polym. Phys., 18, 793 (1980).

8. S. Fujimoto, Kobunshi, 36, 7, 486 (1987).

9. A. Tanaka, H. Sawada, and N. Wakatsuki, Fujitsu Sci. Tech. J., 23(3), 125 (1987).

10. T. Okada and K. Nakamura, Kobunshi, 38, 380 (1989).

11. Nikkei Microdevice, Vol. 10, 1988, p. 54.

12. K. Nagae, J. Hirakata, and S. Kobayashi, Television Gatsukai Tech. Rep., 12, 29 (1988). 